\title{
Achieving Quiescence with Fluocinolone Implants
}

\author{
Freia McGregor Andrew D. Dick Tomas Burke \\ Bristol Eye Hospital, Bristol, UK
}

Keywords

Uveitis · Intraocular inflammation · Fluocinolone acetonide

\section{Abstract}

Persistent anterior uveitis causing cystoid macular oedema may necessitate either intraocular or systemic immunosuppression. This case report highlights how a newly licenced treatment, fluocinolone acetonide (Iluvien ${ }^{\circledR}$, Alimera Sciences Ltd., England, UK) achieves quiescence in refractory and steroid-dependent disease and in the presence of an acute relapse.

\section{Introduction}

We present the case of a 65-year-old Caucasian woman who had been treated for bilateral, chronic anterior uveitis with refractory cystoid macular oedema (CME) for 11 years. Osteoporosis and multiple intolerances to systemic immunosuppression meant that treatment options were limited to local steroid treatment.

\section{Case Report}

This patient had undergone extensive investigations to identify an aetiology of uveitis. All investigations were negative except that she was HLA-B27 positive, but there was no spondyloarthropathy clinically. Past ocular history included bilateral pseudophakia (uveitisrelated cataract) and right amblyopia.

Previous attempts to control intraocular inflammation and maintain disease remission had been largely unsuccessful due to difficulties using both corticosteroids and steroidsparing agents. She had multiple co-morbidities which prevented optimal use of systemic steroids, including severe osteoporosis following total abdominal hysterectomy and bilateral

\section{Karger!}


salpingo-oophorectomy 30 years previously, requiring 3 monthly ibandronate infusions. Trials of steroid-sparing immunosuppressive agents including mycophenolate mofetil, methotrexate, and tacrolimus either did not resolve the CME or resulted in unacceptable systemic side effects including gastrointestinal disturbance and cutaneous rashes (shown in Fig. 1,2). Adalimumab resulted in a good initial response over 3 months but was stopped due to development of a widespread skin rash.

In February 2012, her best-corrected visual acuity (BCVA, Early Treatment Diabetic Retinopathy Study letters) was OD: 25 letters and OS: 70 letters. She had bilateral anterior chamber inflammation OD: 2+, OS: $0.5+$ SUN Score [1] and CME OD with posterior capsular opacification. A $700 \mu \mathrm{g}$ intravitreal dexamethasone implant (OZURDEX ${ }^{\circledR}$, Allergan Ltd., Speke, UK) was inserted into the right eye with resolution of CME (central macular thickness: 336-186 $\mu \mathrm{m}$ at 2 months) and improvement in BCVA 52 letters (planned YAG laser posterior capsulotomy was also performed). The left eye was then treated with an intravitreal dexamethasone implant with excellent clinical response; BCVA improving from 35 to 79 letters. Repeated bilateral intravitreal implants controlled subsequent recurrences of intraocular inflammation and CME (shown in Fig. 3), and however, implant administration was required approximately every 3 months to maintain remission (shown in Fig. 4). Short delays between treatments led to an explosive flare in her uveitis, occasionally with hypopyon precluding fundal view, and an associated profound drop in BCVA that could be rescued with both intravitreal dexamethasone or intravenous methylprednisolone when very severe.

After 22 months of regular intravitreal dexamethasone implants to both eyes, and an inability to deliver a safe tolerable systemic treatment with steroid-responsive uveitis, we administered longer acting intravitreal implants. Fluocinolone acetonide $190 \mu \mathrm{g}$ (Iluvien ${ }^{\circledR}$, Alimera Sciences Ltd., England, UK) was not licensed at that time for use in Uveitis; funding for compassionate and physician-led use was granted through NHS Wales (Abertawe Bro Morgannwg University Health Board). With appropriate consent, bilateral sequential fluocinolone implants were inserted in March 2014 (OD, then OS 2 weeks later). There was a rapid clinical response with resolution of hypopyon OS. Remission was mostly maintained for 3 years. After 24 months, there was a mild flare of anterior chamber inflammation in both eyes $(0.5+)$ which responded to a short course of tapering topical steroid eye drops. Thirty-six months following insertion of the fluocinolone

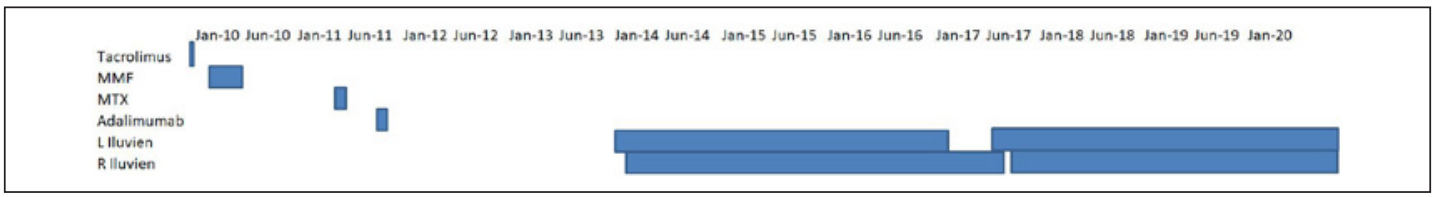

Fig. 1. Timeline of systemic immunosuppression treatments. Outline of the different immunosuppressive used by the patient between 2010 and 2020 and the length of treatment periods. MMF, mycophenolate mofetil; MTX, methotrexate.

\begin{tabular}{|l|l|l|}
\hline \multicolumn{1}{|c|}{ Drug } & \multicolumn{1}{c|}{ Treatment dates } & \multicolumn{1}{c|}{ Reason stopped } \\
\hline Oral Steroids & Intermittent, multiple courses & Contraindication: Severe osteoporosis \\
\hline Tacrolimus & $28 / 01 / 2010-04 / 02 / 2010$ & Intolerant: Gl upset (diarrhoea) \\
\hline $\begin{array}{l}\text { Mycophenolate } \\
\text { mofetil (MMF) }\end{array}$ & $02 / 03 / 2010-20 / 05 / 2010$ & Intolerant: tremor, nausea \\
\hline Methotrexate (MTX) & $31 / 05 / 2011-03 / 06 / 2011$ & Intolerant: GI upset (vomiting) \\
\hline Adalimumab & $27 / 10 / 2011-22 / 12 / 2011$ & Allergy: skin rash, lip swelling, breathlessness \\
\hline
\end{tabular}

Fig. 2. Medication treatment dates and reasons for stopping. Table showing drug names, treatment dates, and the reason why that medication was stopped/unsuitable. MMF, mycophenolate mofetil; MTX, methotrexate. 


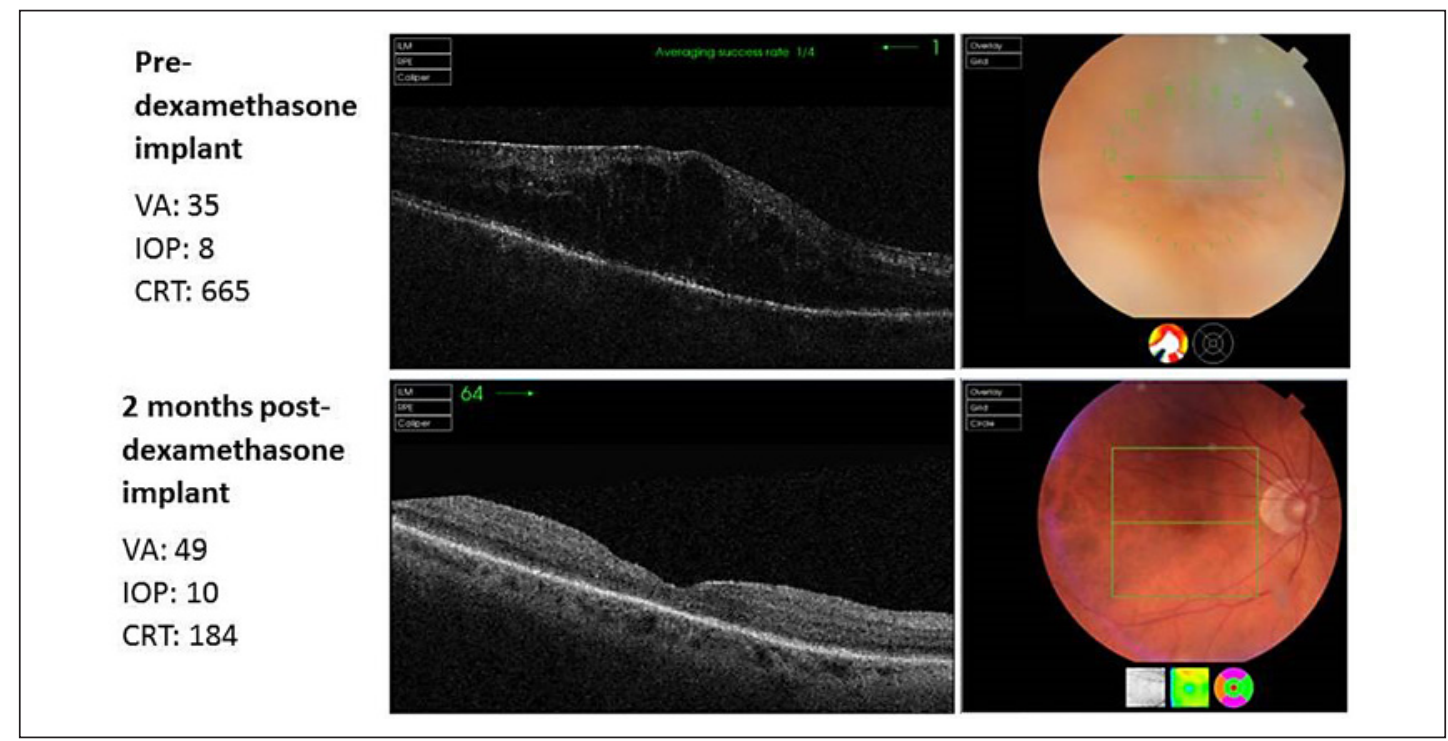

Fig. 3. Clinical response to intravitreal dexamethasone implant right eye (June 2012). Images showing OCT scan and fundus photograph of the right eye before (top image) and after (bottom image) treatment with dexamethasone implant. VA, visual acuity; IOP, intraocular pressure; CRT, central retinal thickness.

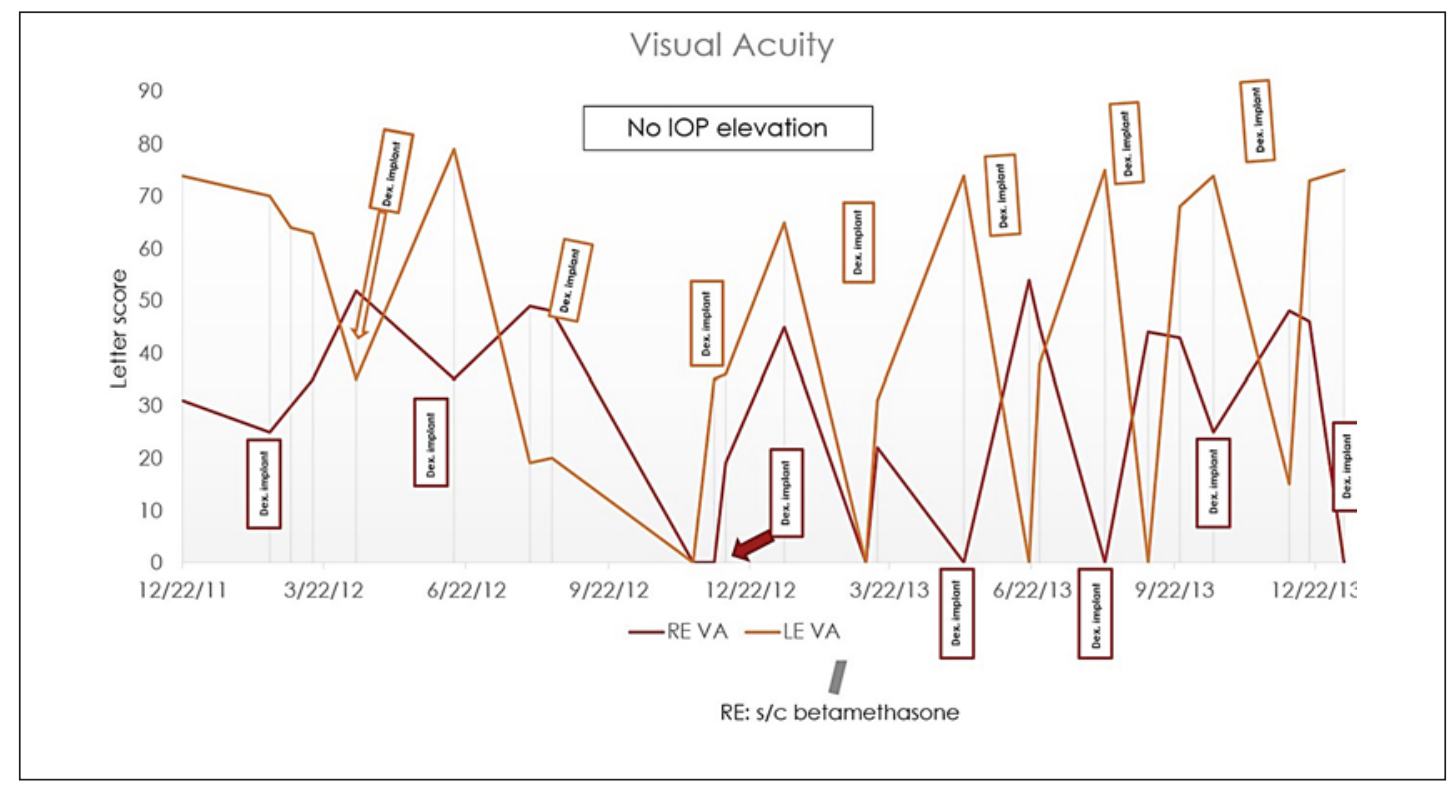

Fig. 4. Clearly shows the ongoing reliance on intravitreal implants and the swift improvement in VA after administration. Right eye in red and left in orange. Graph showing the letter scores over time and the timings of dexamethasone implant administration. IOP, intraocular pressure; VA, visual acuity.

implants, the patient presented with a significant flare of uveitis: hypopyon and significant drop in visual acuity OS. Further intravitreal fluocinolone treatment was administered.

However, there was no improvement in inflammation, and B-scan ultrasonography could not detect the implant in the vitreous cavity. Given concern on the technical delivery that the implant was not inserted, another fluocinolone implant was administered, and inflammation resolved within 2 weeks. An implant was administered OD to maintain quiescence, prior to an anticipated flare of her uveitis. Figure 5 demonstrates the stability of the BCVA after fluocinolone acetonide implant inserted. 


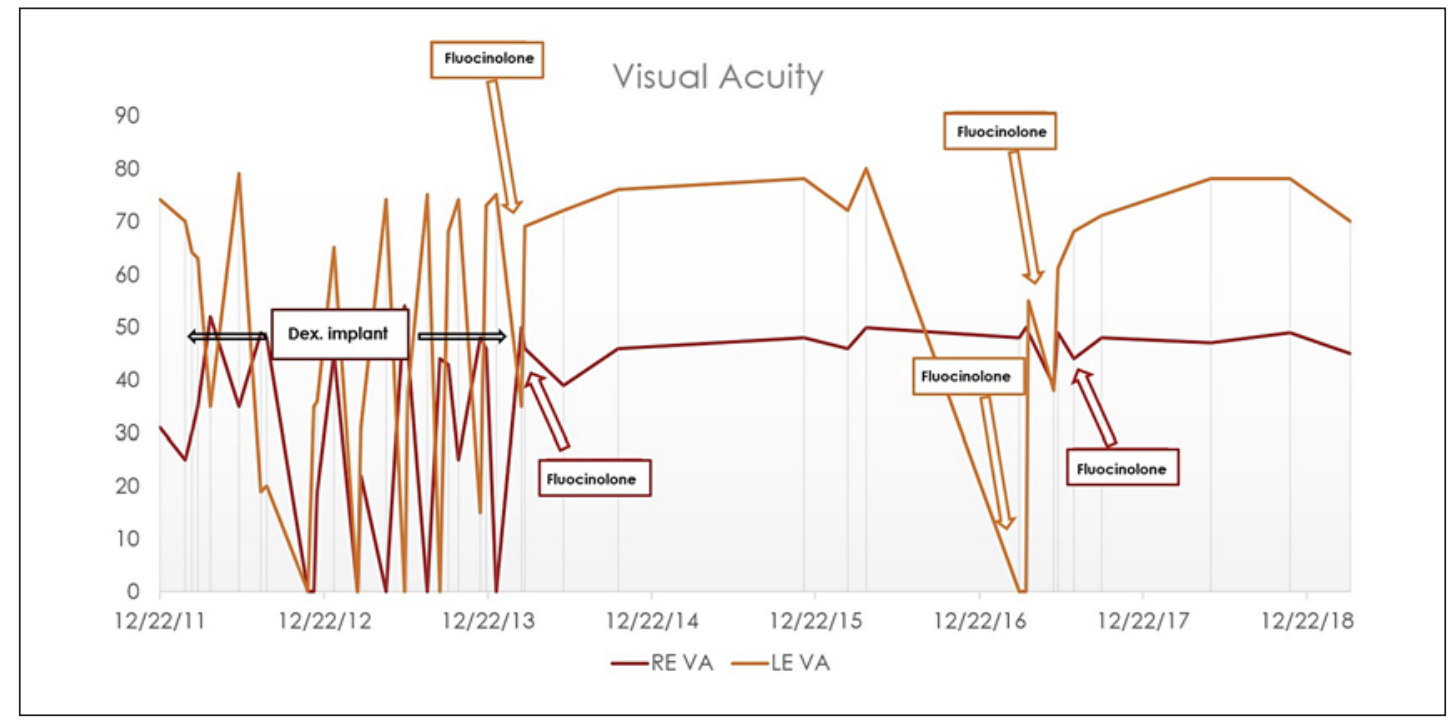

Fig. 5. Demonstrates the stability of VA after fluocinolone implants inserted (including episode where rescue orbital floor triamcinolone was given following failed insertion of implant). Right eye in red and left in orange. VA, visual acuity.

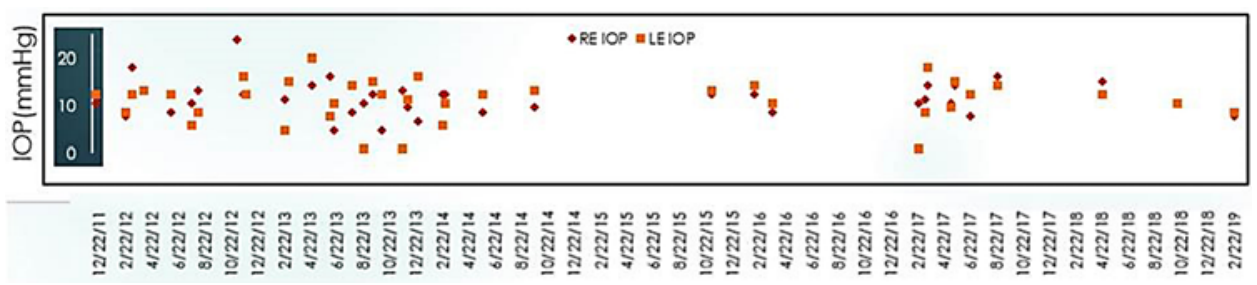

Fig. 6. Shows in the IOP changes over time. Right eye in red and left in orange. This demonstrates the stability of IOP throughout treatment. IOP, intraocular pressure.

During all treatments, there was only 1 IOP rise event (24 mm $\mathrm{Hg}$ ) and that preceded treatment with intravitreal steroid and was associated with a uveitis flare in her right eye. Central retinal thickness (CRT) assessment (TOPCON 3D-OCT 2000) demonstrated resolution of CME. Figure 6 demonstrates the IOP during patient disease course.

\section{Discussion}

Uveitis is a heterogeneous group of conditions characterized by inflammation affecting the uvea (iris, ciliary body, and choroid) [2]. Uveitis incidence has been reported at 52.4 per 100,000 person years [3] and is responsible for causing up to $15 \%$ of cases of preventable blindness in the developed world, mainly in the working-age population [4,5]. The inflammation can be categorized according to location in the eye or by cause (infectious, noninfectious, or masquerade) $[1,6,7]$.

CME is the most common complication of uveitis that results in visual impairment, but other vision-threatening complications include macular scarring, cataract, glaucoma, and hypotony/phthisis [5]. The aim of treatment is to control inflammation and to prevent inflammation-related complications [8]. The mainstay of treatment of noninfectious uveitis is corti- 
costeroids, which can be administered locally (topical, peri-, or intraocular) or systemically $[9,10]$. However, as well as the risk of the disease causing permeant sight loss, side effects associated with treatment can also result in ocular complications, primarlity including glaucoma and cataracts. These cause significant burden on both the patient and the healthcare system $[4,11,12]$. Systemic corticosteroids have additional complications including increased cardiovascular risk, osteoporosis, cushingoid habitus, impaired glucose tolerance and diabetes, and increased susceptibility to infection and mental health and behavioural changes [13].

Fluocinolone acetonide intravitreal implant $190 \mu \mathrm{g}$ (Iluvien ${ }^{\circledR}$ ) is a nonbiodegradable, injectable, corticosteroid implant that releases fluocinolone acetonide at an initial rate of 0.25 $\mu \mathrm{g} /$ day (average rate $0.19 \mu \mathrm{g} /$ day) and is reported to last 36 months [14]. Its use is approved for the prevention of relapse in recurrent noninfectious uveitis affecting the posterior segment of the eye $[15,16]$.

In this case report, the disease course demonstrated that inadequate treatment of intraocular inflammation and CME would lead to irreversible visual loss, and so, alternative treatments were required $[17,18]$. Given the intolerance to, and unsuitability of systemic medication, local corticosteroids were considered most appropriate. By using a longer acting steroid implant, this case demonstrates that it is possible to reduce the burden of intravitreal interventions while maintaining disease control.

The case additionally highlights an ability to maintain remission for 3 years and also a rapid onset of action in face of relapses with hypopyon uveitis, and challenges a general consensus that Iluvien implants have a slow onset of action and may not be appropriate when rapid control of inflammation is required [19]. The minor relapse which occurred reflected the findings of the trials data where $33.3 \%$ of the treatment group had 1 relapse [20]. Jaffe and Pavesio [20] also observed that the time to first recurrence in eyes treated with fluocinolone implants was significantly longer compared to sham-treated eyes. In this case, the relapse was easily controlled highlighting the ability of the implant to modify disease behaviour.

This patient had multiple implants inserted over time to control their disease. While the use of multiple fluocinolone implants has not been studied in uveitic patients, the FAME trials in diabetic macular oedema showed no clinically significant differences in reported outcomes in patients with multiple identical implants inserted $(\geq 3$ implants given in $5.3 \%$ of eyes with DME) [21]. NICE reflect this in their guidance stating in clinical practise patients who have no contraindications are likely to benefit from retreatment when effectiveness of initial implant begins to decline [15].

\section{Conclusion}

This case serves to highlight a use of the newly approved fluocinolone intravitreal implants to help rapidly control CME associated with recurrent noninfectious uveitis. Furthermore, the case demonstrates that such an approach was safe and effective and potentially can be extended to selected patients where systemic treatment is not tolerated and/or effective beyond the July 2019 National Institute for Health and Care Excellence (NICE) approved treatment for the prevention of relapse in recurrent noninfectious uveitis affecting the posterior segment of the eye [15] in the UK. 


\section{Acknowledgement}

Chris Wright, Senior Director Scientific Communications Alimera Sciences Limited review and funding for publication.

\section{Statement of Ethics}

Written consent was obtained from the patient for publication of the case and associated images.

\section{Conflict of Interest Statement}

The authors have no conflicts of interest to declare.

\section{Funding Sources}

Chris Wright, Senior Director Scientific Communications Alimera Sciences Limited review and funding for publication.

\section{Author Contributions}

All authors attest that they meet the current ICMJE criteria for authorship. T.B. and A.D.D. were involved in direct clinical care of the patient. T.B. and F.M. collated the data. F.M. wrote the case report and is acting as corresponding author for submission. T.B., A.D.D, and F.M. were involved in final approval of the version to be published.

\section{References}

1 Jabs DA, Nussenblatt RB, Rosenbaum JT; Standardization of Uveitis Nomenclature (SUN) Working Group. Standardization of uveitis nomenclature for reporting clinical data. Results of the First International Workshop. Am J Ophthalmol. 2005;140(3):509-16.

2 Denniston AK, Nida Sen H.VISUALising a new framework for the treatment of uveitis. Lancet. 2016;388(10050): 1134-6.

3 Gritz DC, Wong IG. Incidence and prevalence of uveitis in Northern California: the Northern California Epidemiology of Uveitis Study. Ophthalmology. 2004;111(3):491-500.

4 Dick AD, Tundia N, Sorg R, Zhao C, Chao J, Joshi A, et al. Risk of ocular complications in patients with noninfectious intermediate uveitis, posterior uveitis, or panuveitis. Ophthalmology. 2016;123(3):655-62.

5 Tomkins-Netzer O, Talat L, Bar A, Lula A, Taylor SRJ, Joshi L, et al. Long-term clinical outcome and causes of vision loss in patients with uveitis. Ophthalmology. 2014;121(12):2387-92.

6 Brady CJ, Villanti AC, Law HA, Rahimy E, Reddy R, Sieving PC, et al. Corticosteroid implants for chronic noninfectious uveitis. Cochrane Database Syst Rev. 2016;2(2):CD010469.

7 Willermain F, Rosenbaum JT, Bodaghi B, Rosenzweig HL, Childers S, Behrend TT, et al. Interplay between innate and adaptive immunity in the development of non infectious uveitis. Prog Retin Eye Res. 2012;31(2):182-94.

8 Gallego-Pinazo R, Dolz-Marco R, Martínez-Castillo S, Arévalo JF, Díaz-Llopis M. Update on the principles and novel local and systemic therapies for the treatment of non-infectious uveitis. Inflamm Allergy Drug Targets. 2013;12:38-45.

9 Haupert CL, Jaffe GJ, Glenn J. New and emerging treatments for patients with uveitis. Int Ophthalmol Clin. 2000;40(2):205-20.

10 Saincher SS, Gottlieb C. Ozurdex (dexamethasone intravitreal implant) for the treatment of intermediate, posterior, and panuveitis: a systematic review of the current evidence. J Ophthalmic Inflamm Infect. 2020;10(1):1. 
McGregor et al.: Achieving Quiescence with Fluocinolone Implants

11 VanderBeek BL, Bonaffini SG, Ma L. The association between intravitreal steroids and post-injection endophthalmitis rates. Ophthalmology. 2015;122(11):2133-315.

12 Chu DS, Johnson SJ, Mallya UG. Healthcare costs and utilization for privately insured patients treated for noninfectious uveitis in the USA. J Ophthalmic Inflamm Infect. 2013;3:64.

13 Stanbury RM, Graham EM. Systemic corticosteroid therapy: side effects and their management. BrJ Ophthalmol. 1998;82(6):704-8.

14 Syed YY. Fluocinolone acetonide intravitreal implant $0.19 \mathrm{mg}$ (ILUVIEN ${ }^{\circledR}$ ): a review in diabetic macular edema. Drugs. 2017:575-83.

15 National Institute for Health and Care Excellence. Fluocinolone acetonide intravitreal implant for treating recurrent non-infectious uveitis. Technology appraisal guidance [TA590]. London, UK: National Institute for Health and Care Excellence; 2019 Jul 31.

16 Alimera Sciences Limited. ILUVIEN 190 micrograms intravitreal implant in applicator SmPC. 2019. Available from: https://www.medicines.org.uk/emc/medicine/27636\#gref. Accessed 15 Oct 2020.

17 Writing Committee for the Multicenter Uveitis Steroid Treatment (MUST) trial and Follow-up Study Research Group, Kempen JH, Altaweel MM, Holbrook JT, Sugar EA, Thorne JE, et al. Association between long-lasting intravitreous fluocinolone acetonide implants vs. systemic anti-inflammatory therapy and visual acuity at 7 years among patients with intermediate, posterior or panuveitis. JAMA. 2017;317(19):1993-2005.

18 Thorne JE, Sugar EA, Holbrook JT, Burke AE, Altaweel MM, Vitale AT, et al. Periocular triamcinolone vs. intravitreal triamcinolone vs. intravitreal dexamethasone implant for the treatment of uveitic macular edema (POINT trial). Ophthalmology. 2019;126(2):283-95.

19 Augustin AJ, Bopp S, Fechner M, Holz F, Sandner D. Three-year results from the Retro-IDEAL study: real-world data from diabetic macular edema (DME) patients treated with ILUVIEN ${ }^{\circledR}(0.19 \mathrm{mg}$ fluocinolone acetonide implant). Eur J Ophthalmol. 2019;1-10.

20 Jaffe GJ, Pavesio CE. Effect of a fluocinolone acetonide insert on recurrence rates in noninfectious intermediate, posterior or panuveitis. Ophthalmology. 2020;127(10):1395-404.

21 Campochiaro PA, Brown DM, Pearson A, Ciulla T; FAME Study Group. Long-term benefit of sustained-delivery fluocinolone acetonide vitreous inserts for diabetic macular edema. Ophthalmology. $2011 \mathrm{Apr} ; 118(4)$ :62635 . 